\title{
SOLUTION OF THE NON-HOMOGENEOUS HELMHOLTZ EQUATION FOR OPTICAL GRATINGS WITH PERFECTLY CONDUCTING BOUNDARIES
}

\author{
By R. P. McClellan and G. W. Stroke
}

1. Introduction. Considerable interest in the exact solution of the problem of diffraction of plane electromagnetic waves by optical gratings and in the attainment of high grating efficiencies has arisen, following the attainment of highresolution gratings, notably those ruled according to the interferometric control principle first described by G. R. Harrison and G. W. Stroke.1, 2, 3

In the late 1950's, following in particular work by R. P. Madden and J. Strong ${ }^{4}$ it became apparent that the scalar wave solutions based on physical optics failed to even approximate a satisfactory solution of the distribution of diffracted energy, among the orders in blazed gratings, especially because of polarization effects. The need for seeking rigorous electromagnetic boundary value solutions, for two orthogonal polarizations $\left(\mathrm{E}_{\|}, \mathrm{E}\right.$ parallel to the groove length, and $\mathbf{H}_{\|}, \mathbf{H}$ parallel to the groove length) in the incident field, was again formally stressed by Maréchal and Stroke, ${ }^{5}$ following a continued awareness of the electromagnetic nature and origins of the diffraction of light by gratings, at least since Lord Rayleigh's work in $1907,{ }^{6}$ and indeed earlier, as far back as Fraunhofer in $1822 .{ }^{7}$

Lord Rayleigh proposed a method of solution, in which it is assumed that the discrete spectrum of diffracted waves, including the infinity of evanescent waves (in each polarization), together with the incident wave is sufficient to satisfy the boundary condition on the surface of the grating. In fact, he obtained a solution of this type, in a closed form (an infinite system of equations with an infinite number of unknowns, the infinity resulting from the infinity of evanescent waves) for a perfectly conducting sinusoidal profile grating, for the $\mathbf{E}_{\|}$ waves. Rayleigh's solution was extended to the $\mathbf{H}_{\mathbb{q}}$ wave, for the sinusoidal grating, by Stroke. ${ }^{8}$ The "Rayleigh" method of solution, was further extended to dielectric gratings, and gratings of a general periodic profile by Bousquet, Petit, Janot, and Hadni. ${ }^{9-14}$

The criterion for attaining the high efficiencies which are sought in optical gratings was first formally given by Stroke $e^{15}$ in 1963 . High efficiencies in optical gratings can only be attained to the extent that polarization is avoided in the diffracted light, when the grating is illuminated in non-polarized light. ${ }^{15}$ Mathematically, the criterion requires that the energy distribution in the diffracted light, for both polarizations, be everywhere the same, as a function of angles of diffraction, for given angles of incidence and wavelengths.

With this criterion and the availability of electronic computers, it rapidly became apparent that $t^{15-22}$ the Rayleigh assumption for the boundary condition could not be physically justified in a general case and that the solution of the grating diffraction problem had to be obtained according to the well-known boundary-solution methods using non-homogeneous Helmholtz equations, ${ }^{23},{ }^{24}$ where no simplifying boundary-condition assumptions are required. 
One form of solution for a perfectly reflecting, quasiplanar surface of infinite spatial extent, and its particularization to periodic surfaces was given by Wir$\operatorname{gin}^{20}$ in 1964, who noted, in conclusion, that the solution, while "exact", appeared in what seemed to be too complicated a form for the required numerical solution.

More recently, Petit ${ }^{21,}{ }^{22}$ obtained a Helmholtz equation solution, in a form readily suitable for numerical computation, and in good agreement with experiment, for the case of a perfectly conducting grating, of a general periodic profile, for the special case of $\mathbf{E}_{\|}$.

In this paper: 1) we give some further justification for the formalism of the non-homogeneous Helmholtz equation solution, with the aid of the theory of distributions, ${ }^{24}$ and, 2) we extend the solution to the case of $\mathbf{H}_{\|}$with perfectly conducting boundaries.

2. $\mathbf{H}_{\|}$Polarization-Normal Incidence. Consider a diffraction grating whose surface is a perfect conductor. Let the periodic surface of the grating be described by the equation $y=f(x)$ where $f$ is a periodic function with period $d$. The coordinate system is chosen with the grating grooves parallel to the $z$-axis and normal to the $x y$ plane. A plane, monochromatic wave is normally incident on the $x z$ plane and is polarized with the $\mathbf{H}$ vector parallel to the $z$ axis. Such an incident wave has the representation

$$
\mathrm{H}^{i}=e^{-i k y}
$$

where $k=2 \pi / \lambda$ and $\lambda$ is the wavelength of the incident wave

For the above geometry the magnetic field vectors of both the incident and diffracted fields have null $x$ and $y$ components. Therefore $\mathbf{H}^{i}$ and $\mathbf{H}^{d}$ will henceforth denote the $z$ components of the magnetic field vectors of the incident and diffracted fields respectively.

The total magnetic field $\mathbf{H}^{t}=\mathbf{H}^{i}+\mathbf{H}^{d}$ must satisfy the following conditions:

(1) $\Delta \mathbf{H}^{t}+k^{2} \mathbf{H}^{t}=0$ for $y>f(x)$

(2) radiation condition for $y \rightarrow \infty$

(3) boundary condition: $\mathbf{n} \cdot \nabla \mathbf{H}^{t}=\partial \mathrm{H}^{l} / \partial n=0$ for $y=f(x)$

where $\mathbf{n}$ is the unit vector normal to the grating surface. For the analysis which follows it is convenient to consider a set of conditions equivalent to those described above. Euclidean three-space may be taken as composed of two regions; (1) the free space region above the grating and (2) the grating region. Region (1) then contains the source of the incident plane wave. It is possible ${ }^{27}$ to consider the diffracted field as produced by "polarization currents" on the surface of the grating. Then the diffracted field satisfies the homogeneous Maxwell equations in region (1) but not on the surface $y=f(x)$. Thus, an equivalent set of conditions to those above is obtained by replacing condition (1) with:

$$
\Delta \mathbf{H}^{d}+k^{2} \mathbf{H}^{d}=0 \text { for } y>f(x) .
$$

In region (1) and on the surface $y=f(x)$ the diffracted field satisfies the non- 
homogeneous Maxwell equations ${ }^{24}$

$$
\nabla \times \mathbf{E}+i \omega \mu \mathbf{H}=\mathbf{M}, \quad \nabla \times \mathbf{H}-(i \omega \epsilon+\sigma) \mathbf{E}=\mathbf{J}
$$

where $\mathbf{M}$ and J are magnetic and electric current densities on the surface $y=f(x)$. These currents are distributions in the sense of Schwartz ${ }^{29}$ and Bouix ${ }^{24}$ and may be regarded as the sources of the discontinuities in $\mathrm{E}$ and $\mathrm{H}$ across the grating surface. The nonhomogeneous Maxwell equations give rise to the following nonhomogeneous Helmholtz equations: $:^{24}$

$$
\begin{aligned}
& \Delta \mathbf{E}+k^{2} \mathbf{E}=i \omega \mu \mathrm{J}-\nabla \times \mathbf{M}+(i \omega \mu)^{-1} \operatorname{grad} \operatorname{div} \mathbf{M} \\
& \Delta \mathbf{H}+k^{2} \mathbf{H}=(i \omega \epsilon+\sigma) \mathbf{M}+\nabla \times \mathbf{J}-(i \omega \mu)^{-1} \operatorname{grad} \operatorname{div} \mathbf{J}
\end{aligned}
$$

Eq. (5) will be solved for $\mathrm{H}^{d}$, while eq. (4) has been solved by Petit ${ }^{(21)}$ for $\mathrm{E}^{d}$ in the case of the $\mathbf{E}_{\llbracket}$ polarization. Because the field $\mathbf{H}^{d}$ has only a $z$ component, eq. (5) reduces to a scalar equation. The right-hand side of (5) further reduces to merely the $z$ component of $\nabla \times \mathrm{J}$. The $\mathbf{M}$ and grad div $\mathbf{J}$ terms drop out because they have null $z$ components.

It follows from equations (2) to (5) and from the preceding remarks that $\mathrm{J}$ may be represented as a distribution in the following way [for example, see Born and $W o l f^{28}$ p. 5 and Petit $^{21}$.]

$$
\mathbf{J}=\mathbf{T} \gamma(x) \delta(y-f(x))
$$

where $\mathbf{T}=$ unit vector tangent to $y=f(x), \gamma(x)$ is some unknown periodic function with period $d$, and $\delta$ is the Dirac delta function. Computing the $z$ component of $\nabla \times \mathrm{J}$ and using properties of distributions (Schwartz ${ }^{29}$ ) it follows that for the subsequent analysis $(\nabla \times \mathrm{J})_{z \text { comp }}$ may be assumed to have the representation

$$
2 i k \phi(x) \delta(y-f(x))
$$

where $\phi(x)$ is another unknown function of period $d$. Thus we may represent the right-hand side of (5) in the same way as Petit ${ }^{21}$ has represented the right-hand side of (4) in solving the problem for the $E_{11}$ polarization. From this point on the solution for the $\mathbf{H}_{11}$ case is identical to Petit's for the $E_{11}$ case except for the different boundary condition used.

The Helmholtz equation to be solved for $\mathrm{H}^{d}$ is

$$
\Delta \mathbf{H}^{d}+k^{2} \mathbf{H}^{d}=2 i k \phi(x) \delta(y-f(x))
$$

which is a simpler form of eq. (5). $\mathrm{H}^{d}(x, y)$ is periodic in $x$ with period $d$, i.e.

$$
\mathbf{H}^{d}(x, y)=\mathbf{H}^{d}(x+d, y)
$$

Because of its periodicity $\mathbf{H}^{d}(x, y)$ may be expressed in the following form after Fourier expansion:

$$
H^{d}(x, y)=\sum_{n=-\infty}^{\infty} H_{n}^{d}(y) e^{i n K x}
$$

where $K=2 \pi / d$. The solution for $\mathrm{H}^{d}$ is formulated in terms of $\phi(x)$. Identifying 
Fourier coefficients of both sides of equation (8), yields

$$
\frac{d^{2} H_{n}^{d}}{d y^{2}}+X_{n}^{2} H_{n}^{d}=\frac{i k K}{\pi} \int_{0}^{d} \phi(x) \delta(y-f(x)) e^{-i n K x} d x
$$

where $X_{n}=\sqrt{k^{2}-n^{2} K^{2}}$.

Petit $^{21}$ has given the solution of this differential equation [in his case for $E_{n}^{d}(y)$ ] in the form

$$
H_{n}^{d}(y)=\frac{k K}{2 \pi X_{n}} \int_{0}^{d} \phi\left(x^{\prime}\right) \exp \left[-i n K x^{\prime}+i X n|y-f(x)|\right] d x^{\prime}
$$

where $x^{\prime}$ is a dummy variable.

Now the boundary condition may be introduced in order to obtain a solution for $\phi(x)$ which will in turn specify $\mathbf{H}^{d}(x, y)$. The boundary condition has the alternate forms given by the equation

$$
\frac{\partial H^{t}}{\partial n}=\frac{\partial H^{i}}{\partial n}+\frac{\partial H^{d}}{\partial n}=\mathbf{n} \cdot \nabla \mathbf{H}^{t}=0
$$

for $y=f(x)$, where

$$
\mathbf{n}=\frac{-f^{\prime}(x)}{\sqrt{1+f^{\prime}(x)^{2}}} \mathbf{i}+\frac{1}{\sqrt{1+f^{\prime}(x)^{2}}} \mathbf{j}
$$

Evaluating the individual terms of eq. (14), substituting and interchanging the order of integration and summation, eq. (14) becomes

$$
\begin{aligned}
& \int_{0}^{d} \sum_{n=-\infty}^{\infty} \frac{k K}{2 \pi}\left[\frac{X_{n} \operatorname{sgn}\left(f(x)-f\left(x^{\prime}\right)\right)-f\left(x^{\prime}\right) n K}{k X_{n}}\right] \\
& \cdot \phi\left(x^{\prime}\right) \exp \left[i n K\left(x-x^{\prime}\right)+i X_{n}\left|f(x)-f\left(x^{\prime}\right)\right|\right] d x^{\prime}=e^{-i k f(x)}=g(x)
\end{aligned}
$$

Now in eq. (16) let

$$
\begin{aligned}
& S\left(x, x^{\prime}\right)=\frac{k K}{2 \pi} \sum_{n=-\infty}^{\infty} {\left[\frac{X_{n} \operatorname{sgn}\left(f(x)-f\left(x^{\prime}\right)\right)-f\left(x^{\prime}\right) n K}{k X_{n}}\right] } \\
& \cdot \exp \left[i n K\left(x-x^{\prime}\right)+i X_{n}\left|f(x)-f\left(x^{\prime}\right)\right|\right]
\end{aligned}
$$

Eq. (16) then becomes

$$
\int_{0}^{d} \phi\left(x^{\prime}\right) S\left(x, x^{\prime}\right) d x^{\prime}=g(x)
$$

which is recognized as a Fredholm equation of the first kind.

The Fredholm eq. (18) may be solved ${ }^{21}$ by expanding both sides of eq. (18) in Fourier series and equating corresponding Fourier coefficients of the two sides.

If $\phi\left(x^{\prime}\right)$ and $g(x)$ have the Fourier series representations

$$
\phi\left(x^{\prime}\right)=\sum_{j^{\prime}=-\infty}^{\infty} \phi_{j^{\prime}} e^{i j^{\prime} K x^{\prime}} \quad g(x)=\sum_{j^{\prime}=-\infty}^{\infty} g_{j} e^{i j K x}
$$


and setting

$$
S_{j j^{\prime}}=\int_{0}^{d} \int_{0}^{d} S\left(x, x^{\prime}\right) e^{i K\left(j^{\prime} x^{\prime}-j x\right)} d x d x x^{\prime}
$$

then eq. (18) may be solved for $\phi\left(x^{\prime}\right)$ by solving the system of equations

$$
\sum_{j=-\infty}^{\infty} S_{j j}{ }^{\prime} \phi_{j}{ }^{\prime}=g_{j}
$$

Eq. (22) gives the solution of the $\mathbf{H}_{11}$ boundary value problem, for the perfectly conducting grating in normal incidence.

3. $\mathbf{H}_{\Downarrow}$ Polarization-General Angle of Incidence. We now generalize the solution of Section 2, for all angles. The incident wave has the following form

$$
\mathbf{H}^{i}=e^{i k(x \sin \theta-y \cos \theta)}
$$

The diffracted field $\mathrm{H}^{d}$ obeys the relation.

$$
\mathrm{H}^{d}(x+d, y)=\alpha \mathbf{H}^{d}(x, y)
$$

where $\alpha$ is a phase change introduced by the lack of symmetry due to an oblique angle of incidence. Eq. (14) also applies here. Using eq. (23) and eq. (15), eq. (14) yields.

$$
\left[\frac{f(x) i k \sin \theta}{\sqrt{1+f^{\prime}(x)^{2}}}+\frac{i k \cos \theta}{\sqrt{1+f^{\prime}(x)^{2}}} \mathbf{H}^{i}\right]=\frac{-f(x) \partial \mathrm{H}^{d} / \partial x}{\sqrt{1+f^{\prime}(x)^{2}}}+\frac{\partial \mathrm{H}^{d} / \partial y}{\sqrt{1+f^{\prime}(x)^{2}}}
$$

Since eq. (25) must hold for $x+d$ as well as for $x$ and since

$$
\mathbf{H}^{i}(x+d)=\mathbf{H}^{i}(x) e^{i k d \sin \theta}
$$

it is clear that

$$
\alpha=e^{i k d \sin \theta}
$$

This gives the following expressions for $\mathbf{H}^{d}(x, y)$.

$$
\mathbf{H}^{d}(x, y)=\exp (i k x \sin \theta) \sum_{n=-\infty}^{\infty} H_{n}^{d}(y) e^{i n K x}
$$

It may be easily verified by substitution that eq. (28) satisfies the condition of eq. (24). Eq. (28) corresponds to eq. (10).

Following the same procedure used in section (2), $\mathrm{H}_{n}^{d}$ must satisfy the following differential equation.

$$
\frac{d^{2} H_{n}^{d}}{d y^{2}}+X_{n}^{2} H_{n}^{d}=\frac{i k K}{\pi} \int_{0}^{d} \Gamma(x) \delta(y-f(x)) e^{-i n K x} d x
$$

where now

$$
X_{n}=\sqrt{k^{2}-(n K+k \sin \theta)^{2}}
$$

and $\Gamma(x)$ is an unknown periodic function of period $d$. 
The boundary condition yields the following equation.

$$
\begin{gathered}
\int_{n=-\infty}^{d} \frac{k K}{2 \pi}\left[\frac{X_{n} \operatorname{sgn}\left(f(x)-f\left(x^{\prime}\right)\right)-f^{\prime}(x)(n K+k \sin \theta)}{k X_{n}}\right] \Gamma\left(x^{\prime}\right) \\
\cdot \exp \left[i n K\left(x-x^{\prime}\right)+i X_{n}\left|f(x)-f\left(x^{\prime}\right)\right|\right] d x^{\prime} \\
=\left[\cos \theta+f^{\prime}(x) \sin \theta\right] \exp [i k(-f(x) \cos \theta)]=h(x)
\end{gathered}
$$

Next letting

$$
\begin{array}{r}
T\left(x, x^{\prime}\right)=\frac{k K}{2 \pi} \sum_{n=-\infty}^{n}\left[\frac{X_{n} \operatorname{sgn}\left(f(x)-f\left(x^{\prime}\right)\right)-f^{\prime}(x)(n K+k \sin \theta)}{k X_{n}}\right] \\
\cdot \exp \left[i n K\left(x-x^{\prime}\right)+i X_{n}\left|f(x)-f\left(x^{\prime}\right)\right|\right]
\end{array}
$$

eq. (31) reduces to

$$
\int_{0}^{d} \Gamma\left(x^{\prime}\right) T\left(x, x^{\prime}\right) d x^{\prime}=h(x)
$$

which may be solved in the same way as eq. (18).

4. Diffracted Field Above Grating Surface. It may be of interest to note the form of $\mathrm{E}^{d}$ and $\mathrm{H}^{d}$ for $y>\max [f(x)]$, that is the form of the diffracted field above the grating.

$\mathrm{H}^{d}$ of eq. (28), has the form

$$
\mathrm{H}^{d}(x, y)=\sum_{n=-\infty}^{\infty} B n e^{i\left(k x \sin \theta+n K x+x_{n} y\right)}
$$

where

$$
B n=\frac{k K}{2 \pi X n} \int_{0}^{d} \Gamma\left(x^{\prime}\right) e^{-i n K x^{\prime}-i X n f\left(x^{\prime}\right)} d x^{\prime}
$$

We also recall, according to Petit ${ }^{21}$ that $\mathrm{E}^{d}$ has the form

$$
\mathbf{E}^{d}(x, y)=\sum_{n=-\infty}^{\infty} A_{n} e^{i\left(k x \sin \theta+n K x+x_{n} y\right)}
$$

where

$$
A n=\frac{k K}{2 \pi X n} \int_{0}^{d} \Phi\left(x^{\prime}\right) e^{-i n K x^{\prime}-i X n f\left(x^{\prime}\right)} d x^{\prime}
$$

5. Numerical Calculations. Petit ${ }^{21}$ has described a procedure for computing approximate solutions for the system of eqs. (22). The approximate solution consists of some finite number $F$ of the Fourier components of $\phi(x)$ (i.e. a finite sum of terms form the right-hand side of eq. (19)). To obtain these Fourier components we solve a $F \times F$ subsystem of the system of eqs. (22). For each of the $S_{j j^{\prime}}$, coefficients in this subsystem, we will use an $N$-term approximation obtained by consideration of eq. (17) and eq. (21).

Details will be given when a program has actually given results in a form comparable to our experimental measurements. For an independently obtained 
solution of the $\mathbf{H}_{\|}$problem involving a somewhat more elaborate approach than that which we present (and requiring renormalization), which has appeared after our above solution was first submitted, the reader may also wish to consult our reference $(30)$.

6. Conclusion. We may finally note that we have good experimental evidence to indicate that multifacet grooves (consisting, for example, of three planefacetted sections) give higher efficiencies, through a broader angular range, than grooves with two-facet saw-tooth cross-sections.

Acknowledgment. One of us (GWS) wishes to acknowledge several fruitful conversations held with $\mathrm{R}$. Petit over the course of several years, in connection with this work, as well as a conversation with $\mathrm{A}$. Wirgin in early stages of Wirgins work (ref. 20), and many fruitful conversations and kind interest by A. Maréchal

This paper reports in part work carried out under Grant NsG-698 from the National Aeronautics and Space Administration.

\section{BIBLIOGRAPHY}

1. G. R. Harrison and G. W. Stroke, "Interferometric Control of Grating Ruling with Continuous Carriage Advance", J. Opt. Soc. Am. 45, 112 (1955).

2. G. R. Harrison, "The Controlled Ruling of Diffraction Gratings", Proc. American Philos. Soc. 120, 483 (1958).

3. G. W. Stroke, "Attainment of High-Resolution Gratings By Ruling under Interferometric Control", J. Opt. Soc. Am. 51, 1321 (1961).

4. R. P. Madden and J. Strong, "Diff raction Gratings", in J. Strong, Concepts of Classical Optics (W. H. Freeman and Co., San Francisco, 1948).

5. A. Marechal and G. W. Stroke, "Sur l'origine des effets de polarisation et de diffraction dans les réseaux optiques", C.r.Ac.Sc. 249, 2042 (1959).

6. Lord Rayleigh, "Dynamical Theory of Gratings", Proc. Roy. Soc. (London) A79, 399 (1907).

7. For a general background, see e.g. G. W. STRoKE, "Diffraction Gratings" in Handbuch der Physik, Vol. 29, ed. S. Flügge (Springer Verlag, Berlin and Heidelberg, 1965) in print.

8. G. W. Stroke, "Etude Théorique et Expérimentale de deux Aspects de la Diffraction dans les réseaux Optiques. L'évolution des défauts dans les figures de diffraction et l'Origine Electromagnétique de la Répartition entre les Ordres," Rev. Opt. 39, 291 (1960).

9. P. Bousquet, "Etude de la Reflexion et de la Transmission de la Lumière par un Réseau Transparent à Profil Sinusoidal. Extension au Cas d'une Surface Irregulière," Rev. Opt. 41, 277 (1962).

10. P. Bousquet and R. Deleurl, "Diffraction des Ondes Electromagnétiques par un Réseau à Profil Sinusoidal", Compt. Rend. 256, 1461 (1963).

11. P. Bousquet, "Diffraction des Ondes Electromagnétiques par un Réseau à Facettes Planes", Compt. Rend. 256, 3422 (1963).

12. R. Petit, "Contribution à l'Etude de la Diffraction d'une Onde Plane par un Réseau Métallique," Rev. Opt. 42, 263 (1963).

13. P. Bousquet, "Diff raction des Ondes Electromagnétiques par un Réseau à Profil Triangulaire," Compt. Rend. 257, 80 (1963).

14. C. Janot and A. Hadni, "Polarisation de la lumière par les réseaux échelettes dans l'infrarouge lointain," J. Physique 24, 1073 (1963). 
15. G. W. Stroke, "Attainment of High Efficiencies in Blazed Optical Gratings by Avoiding Polarization in the Diff racted Light," Phys. Letters, 6, 45 (1963).

16. R. F. JARRell and G. W. Stroke, "Some New Advances in Grating Ruling, Replication and Testing," Applied Optics, 3, 1251 (1964).

17. V. Twersky, e. g. "On Multiple Scattering of Waves," Report to the XIIIth Gen. Assembly of the Int'l Scientific Radio Union (London, Sept. 5-15, 1960).

18. G. Lippmann, "Note on Theory of Gratings," J. Opt. Soc. Am. 43, 408 (1953)

19. R. Petit, "Contribution à L'Etude de la Diffraction d'une onde Plane par un Réseau Métallique", Revue d'Optique 6, 263 (1963).

20. A. WiRgin, "Considérations Théoriques sur la Diffraction par Réflexion sur des Surface Quasiment Planes; Applications à la Diffraction par les Réseaux", Rev. Optique 43, 449 (1964)

21. R. Petit and M. Cadilhac, "Etude théorique de la diffraction par un réseau", C.r. Ac.Sc. 259, 2077 (1964)

22. R. Petit, "Etude numérique de la diffraction par un réseau", C.r.Ac.Sc. 260, 4454 (1965)

23. P. M. Monse and H. Feshrach, Methods of Theoretical Physics, (McGraw-Hill Book Co., Inc. New York, 1953).

24. M. Bovrx, Les Fonctions Généralisées ou Distributions (Masson et Cie, Paris, 1964)

25. G. W. Stroke, An Introduction to Coherent Optics and Holography (Academic Press, Inc. New Jork and London, 1966).

26. A) A. Sommerfend, Partial Differential Equations in Physics, (Academic Press, Inc. New York, 1949).

B) A. Sommerfeld, in Jahresb. Deutsch. Math. Ver. 21, 326, (1912).

27. S. Schelkonoff, Electromagnetic Waves (Van Nostrand Book, Co., New York, 1943).

28. Born and Wolf, Principles of Optics, (Macmillan Book Co., New York, 1964).

29. L. Schwartz, Theorie des Distributions vol. 1. (Hermann et Cie, Paris, 1950).

30. R. Petst, "Étude théorique de la diffraction pzr un réseau métallique", C.r.Ac.Sc. 261, 4677 (1965).

UNIVERSITY OF Michigan

(Received August 30, 1965; Revised July 20, 1966) 